\title{
DNMT3A and TET1 cooperate to regulate promoter epigenetic landscapes in mouse embryonic stem cells
}

Tianpeng Gu${ }^{1 \dagger}$, Xueqiu Lin ${ }^{4,5,6,10 \dagger}$, Sean M. Cullen ${ }^{1,2,3 \dagger}$, Min Luo ${ }^{1}$, Mira Jeong ${ }^{1}$, Marcos Estecio ${ }^{7}$, Jianjun Shen", Swanand Hardikar ${ }^{7}$, Deqiang Sun ${ }^{4,5,8}$, Jianzhong Su ${ }^{4,5}$, Danielle Rux ${ }^{9}$, Anna Guzman ${ }^{1}$, Minjung Lee ${ }^{8}$, Lei Stanley Qi ${ }^{10}$, Jia-Jia Chen ${ }^{11}$, Michael Kyba ${ }^{9}$, Yun Huang ${ }^{8}$, Taiping Chen ${ }^{7}$, Wei $\mathrm{Li}^{4,5^{*}}$ and Margaret A. Goodell ${ }^{1,2^{*}}$ (D)

\begin{abstract}
Background: DNA methylation is a heritable epigenetic mark, enabling stable but reversible gene repression. In mammalian cells, DNA methyltransferases (DNMTs) are responsible for modifying cytosine to 5-methylcytosine $(5 \mathrm{mC})$, which can be further oxidized by the TET dioxygenases to ultimately cause DNA demethylation. However, the genome-wide cooperation and functions of these two families of proteins, especially at large under-methylated regions, called canyons, remain largely unknown.

Results: Here we demonstrate that DNMT3A and TET1 function in a complementary and competitive manner in mouse embryonic stem cells to mediate proper epigenetic landscapes and gene expression. The longer isoform of DNMT3A, DNMT3A1, exhibits significant enrichment at distal promoters and canyon edges, but is excluded from proximal promoters and canyons where TET1 shows prominent binding. Deletion of Tet 1 increases DNMT3A1 binding capacity at and around genes with wild-type TET1 binding. However, deletion of Dnmt3a has a minor effect on TET1 binding on chromatin, indicating that TET1 may limit DNA methylation partially by protecting its targets from DNMT3A and establishing boundaries for DNA methylation. Local CpG density may determine their complementary binding patterns and therefore that the methylation landscape is encoded in the DNA sequence. Furthermore, DNMT3A and TET1 impact histone modifications which in turn regulate gene expression. In particular, they regulate Polycomb Repressive Complex 2 (PRC2)-mediated H3K27me3 enrichment to constrain gene expression from bivalent promoters.
\end{abstract}

Conclusions: We conclude that DNMT3A and TET1 regulate the epigenome and gene expression at specific targets via their functional interplay.

Keywords: DNMT3A, TET1, DNA methylation, H3K27me3, PRC2, Embryonic stem cells

\section{Background}

DNA methylation at the 5-position of cytosine $(5 \mathrm{mC})$ on CpG dinucleotides is a heritable epigenetic marker in mammals that is critical for development, $\mathrm{X}$-chromosome inactivation, silencing of transposons and repeat elements; aberrant DNA methylation is often implicated in

\footnotetext{
* Correspondence: w11@bcm.edu; goodell@bcm.edu

${ }^{\dagger}$ Tianpeng Gu, Xueqiu Lin and Sean M. Cullen contributed equally to this work.

${ }^{4}$ Division of Biostatistics, Dan L. Duncan Cancer Center, Baylor College of

Medicine, Houston, TX 77030, USA

${ }^{1}$ Stem Cells and Regenerative Medicine Center, Baylor College of Medicine, Houston, TX 77030, USA

Full list of author information is available at the end of the article
}

carcinogenesis $[1,2]$. DNA methylation is generated by de novo methyltransferases $3 \mathrm{~A}$ and $3 \mathrm{~B}$ (DNMT3A and DNMT3B) and maintained by DNMT1. Mouse mutants lacking one or more DNMTs exhibit aberrant development [3, 4]. Dnmt triple knockout (TKO) embryonic stem cells (ESCs) progressively lose differentiation potential [5]. While DNA methylation is generally uniformly high throughout the genome (60-80\% of CpGs), it is largely excluded from some regions, notably promoters, $\mathrm{CpG}$ islands (CGIs) and large under-methylated regions termed canyons (or valleys) [6,7]. In addition, the pattern is broadly stable across the genome, except at certain regions

(C) The Author(s). 2018 Open Access This article is distributed under the terms of the Creative Commons Attribution 4.0 International License (http://creativecommons.org/licenses/by/4.0/), which permits unrestricted use, distribution, and 
such as enhancers [8] and canyon edges [6,9]. The specific contributions of DNMTs to these dynamics and the mechanisms that exclude DNA methylation from certain regions are not well understood.

Ten-eleven translocation (TET) proteins have been identified as dioxygenases that convert $5 \mathrm{mC}$ to 5-hydroxymethycytosine $\quad(5 \mathrm{hmC}), \quad 5$-formylcytosine (5fC), and 5-carboxylcytosine (5caC) [10-12]. $5 \mathrm{mC}$ oxidation coupled with TDG-mediated base excision of $5 \mathrm{fC}$ or $5 \mathrm{caC}$ constitutes an active demethylation pathway [10]. TET proteins play important roles in ESC self-renewal and transcriptional regulation [13-15]. Both TET1 and TET2 are dispensable for embryonic development while TET3 is essential for oocyte reprogramming [16-18]. The roles of TET enzymes in the establishment and maintenance of the global DNA methylation pattern remain an area of intense research.

The genome-wide DNA methylation landscape changes dynamically during mammalian development [19]. Global waves of DNA demethylation mediated by TETs and re-methylation by DNMTs take place during early embryogenesis and gametogenesis. However, whether and how they function together to regulate DNA methylation, especially at specific genomic regions such as CGIs or canyons, has not yet been deeply investigated.

In the present work, we have taken advantage of mouse embryonic stem cells, where both DNMT3A/3B and TET1 are highly expressed, to elucidate the binding behaviors of DNMT3A and TET1 around transcriptional start sites (TSS) or canyons. We demonstrated that DNMT3A and TET1 impact gene expression via alterations in the histone landscapes surrounding these regions. In particular, they regulate gene expression at poised bivalent genes through affecting Polycomb Repressive Complex 2 (PRC2)-mediated H3K27me3 enrichment.

\section{Results \\ Global DNA methylation in mouse ESCs is predominantly regulated by DNMT3A}

To examine the distinct contributions of DNMT3A and DNMT3B to DNA methylation in mouse ESCs, we examined the patterns of DNA methylation after loss of Dnmt3a or Dnmt3b. DNA methylation landscapes at single-base resolution were generated by whole genome bisulfite sequencing (WGBS) between wild type (WT), Dnmt3a KO, and Dnmt3b KO J1 ESCs [3] with similar passage numbers. Over one billion sequencing reads were generated for each cell type, resulting in an average coverage of around 30-fold in each dataset. Although both methyltransferases are highly expressed and are known to contribute to maintenance of methylation genome-wide and at repetitive elements [3], loss of Dnmt3a had a much more dramatic impact than loss of Dnmt3b on DNA methylation genome-wide (Fig. 1a and
Additional file 1: Figure S1a), with a significant decrease of CpG methylation level on distal promoters and at canyon [6] edges (Fig. 1b), as exemplified by the Sox2 locus (Fig. 1c). The Dnmt3a KO ESCs also showed more significant reduction of non-CpG methylation (Additional file 1: Figure S1b). Compared to WT cells, the majority of differentially methylated regions (DMRs) in either $\mathrm{KO}$ cell line were hypomethylated (Additional file 1: Figure S1c), while a very small number of CpGs acquired methylation (Additional file 1: Figure S1d), likely due to aberrant activity of remaining methyltransferases or DNMT1. Consistent with the general hypomethylation, the number of short under-methylated regions (UMRs; $1 \mathrm{~kb} \leq$ length $<3.5 \mathrm{~kb}$, methylation $\leq 10 \%$ ) approximately doubled in Dnmt3a KO cells $(n=22,280)$ compared to both WT $(n=9920)$ and Dnmt3b KO $(n=12,303)$ cells (Fig. 1d). In addition, the number of canyons (UMRs $\geq 3.5 \mathrm{~kb}$ ) quadrupled in Dnmt3a KO cells $(n=3907$, compared to 807 in WT, Fig. 1e). Together, these data demonstrate a predominant role of DNMT3A over DNMT3B in maintaining the global DNA methylation pattern in mouse ESCs.

DNMT3A1 is enriched at distal promoter and canyon edges We next sought to understand the relationship between the DNA methylation pattern and DNMT binding. At least two Dnmt3a and six Dnmt3b isoforms have been identified [20], among them Dnmt3a2 and Dnmt3b1 are catalytically active and strongly expressed in mouse ESCs. Previous work demonstrated DNMT3B1 preferentially binds within gene bodies of actively transcribed genes; both DNMT3A2 and DNMT3B1 are excluded from active promoters and enhancers [21]. The two isoforms of Dnmt3a originate from different promoters and differ by $219 \mathrm{~N}$-terminal amino acids; both retain the catalytically active C-terminus [22]. Although DNMT3A2 predominates in ESCs, the longer isoform DNMT3A1 is still expressed (Fig. 2a). Thus, we explored the occupancy of DNMT3A1 on chromatin, trying to compare and reveal any binding difference between these two isoforms.

To examine the binding activity of DNMT3A1, we developed a Bio-Dnmt3a1 ES cell line in a WT background that allows for doxycycline-inducible in vivo biotinylation of DNMT3A1. The generated cell line expresses an N-terminal Avi-tagged DNMT3A1 under control of a TetO promoter [23]. An IRES-biotin ligase (BirA) sequence included in the vector allows for biotin tagging at the $\mathrm{N}$-terminus of DNMT3A1. After confirming induction of the biotinylated-DNMT3A1 protein (Additional file 1: Figure S2a), genome-wide binding maps of DNMT3A1 were generated by biotin-based chromatin immunoprecipitation (ChIP) followed by high-throughput sequencing. DNMT3A1, and to a lesser 


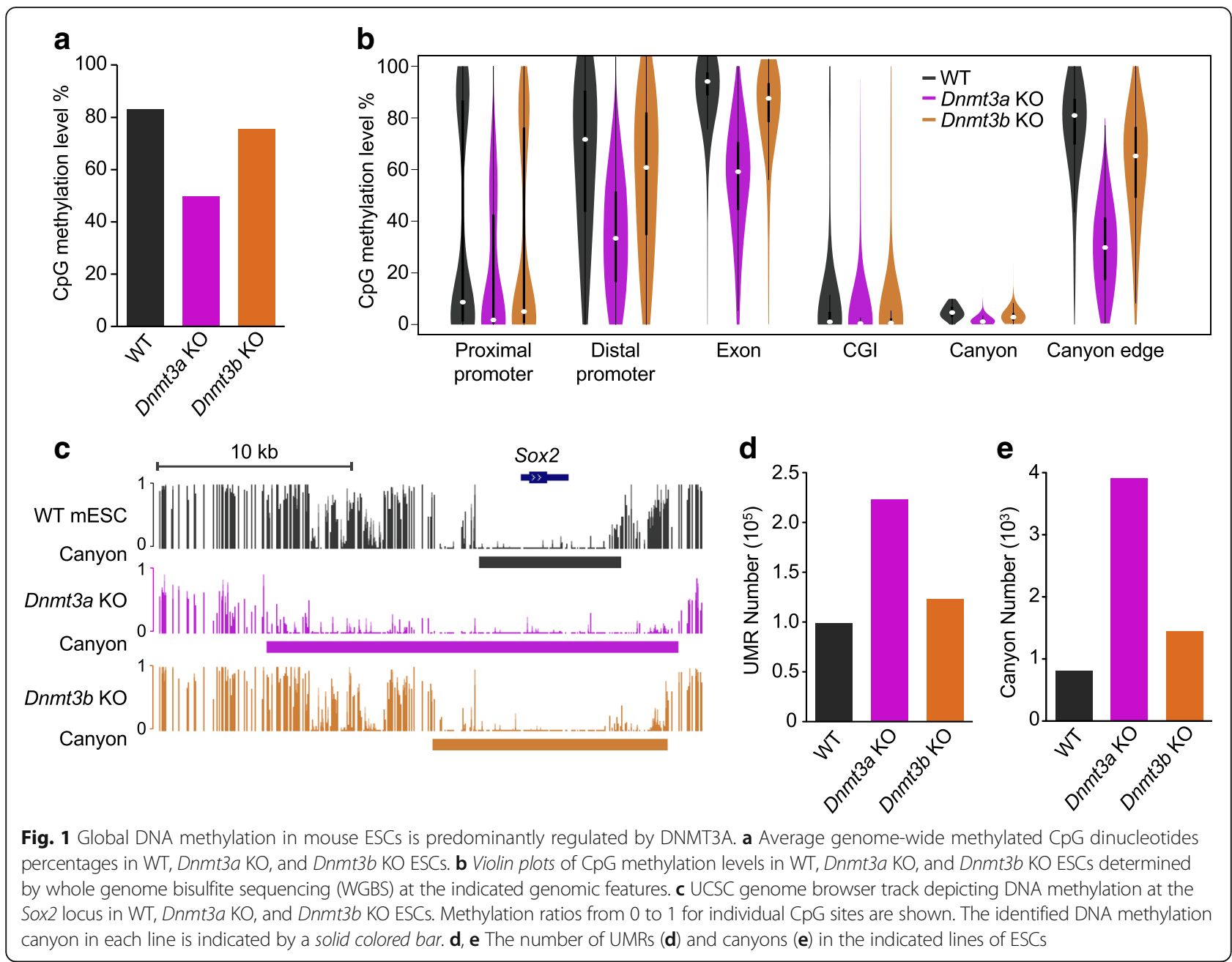

extent DNMT3A2, is preferentially enriched in distal promoter regions (500-3000 bp upstream of TSSs), differing from the specific genic enrichment within exon/ gene bodies, as observed for DNMT3B1 (Fig. 2b and Additional file 1: Figure S2b). Strikingly, DNMT3A1 and
DNMT3A2 are most enriched at canyons edges $(2 \mathrm{~kb}$ flanking both sides of a canyon, Fig. $2 \mathrm{~b}$ and Additional file 1: Figure S2c). DNMT3A2 also binds in gene bodies. All DNMTs, with DNMT3A1 to the largest extent, are excluded from regions known to have low methylation,
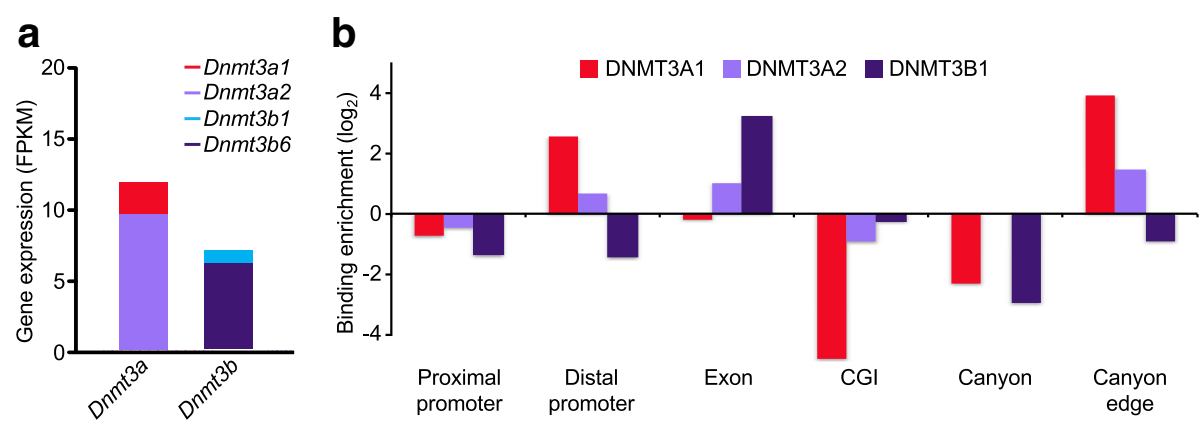

Fig. 2 DNMT3A1 is enriched at distal promoter and canyon edges. a Expression levels (FPKM) determined by RNA-seq of major active isoforms of Dnmt3a: Dnmt3a1 (NM_001271753, NM_007872), Dnmt3a2 (NM_153743), and Dnmt3b: Dnmt3b1 (NM_001003961), Dnmt3b6 (NM_001003963) in WT J1 ESCs. b Relative binding enrichment between DNMTs (DNMT3A1, DNMT3A, and DNMT3B1) at different genomic features, compared to their average genomic distribution. The canyon edge corresponds to 2-kb flanking regions around canyons. DNMT3A2 and DNMT3B1 data are from [21] 
such as proximal promoters (TSS $\pm 500 \mathrm{bp}$ ), CGIs, and canyons themselves (Fig. 2b). It has been reported that both DNMT3A2 and DNMT3B1 prefer methylated CpG-rich regions, with a drop off at CGIs [21]. DNMT3A1 exhibits a trend very similar with DNMT3A2 and DNMT3B1. However, there is more DNMT3A2 and DNMT3B1 binding at regions with higher CpG density where DNMT3A1 appears to drop off sharply (Additional file 1: Figure S2d). Our data suggested that DNMT3A1 has unique properties in genome binding and may have special functions in regulating DNA methylation and gene expression. These clear distinctions led us to focus on the potential roles of DNMT3A1 at distal promoters and canyons.

DNMT3A1 and TET1 have complementary binding patterns We had previously shown in mouse hematopoietic stem cells (HSCs) that $5 \mathrm{hmC}$ accumulates at the edges of canyons; we proposed that the TET family of proteins (TET1/2/3), which catalyze conversion of $5 \mathrm{mC}$ to $5 \mathrm{hmC}$, may be localized to these sites [6]. To test this hypothesis, we examined the binding relationship between DNMT3A1 and TET1, the TET family member most highly expressed in mouse ESCs. To determine the binding pattern of TET1 genome-wide, CRISPR/Cas9-facilitated homology directed repair (HDR) was utilized to develop an ESC line that contains a C-terminal $3 \times$ FLAG tag on both alleles (Tet1-FLAG ESCs, Additional file 1: Figure S3a). Similar $5 \mathrm{mC}$ and $5 \mathrm{hmC}$ levels were observed by dot blot with genomic DNA from WT and Tet1-FLAG ESCs, indicating proper enzymatic activity of TET1-FLAG protein (Additional file 1: Figure S3b-e). We also performed ChIP-seq with Tet1-FLAG ESCs using anti-FLAG M2 and an anti-TET1 antibody [24]. A strong correlation between the two datasets suggested that the C-terminal tag did not interfere with the genome localization of TET1 and suggested we could accurately measure TET1 protein distribution on chromatin (Additional file 1: Figure S4a).

The binding pattern of TET1 was highly complementary to DNMT3A1 at specific genomic regions, particularly canyons and TSSs. TET1 binds throughout canyons, while DNMT3A1, excluded from binding within the canyon itself, exhibits peak binding at the canyon edges (Fig. 3a and Additional file 1: Figure S4b). As exemplified at the Foxo1 locus, TET1 is seen binding in the canyon from edge-to-edge, whereas DNMT3A1 accumulates at the edges and outside the canyon (Fig. $3 \mathrm{~b}$ ). In addition, at TSSs, where TET1 is known to accumulate [14, 15, 24], this opposing binding pattern was similarly observed (Fig. 3c and Additional file 1: Figure S4c). Broadly, these observations are consistent with a reciprocal relationship of these proteins in their ability to interact with DNA containing different levels of CpG density.
Specifically, as $\mathrm{CpG}$ density increases $>1.5 \mathrm{CpG}$ per 100 bp, TET1 binding dramatically increases, while DNMT3A1 binding diverges in the opposite direction (Fig. 3d), suggesting that $\mathrm{CpG}$ density might influence the complementary genomic occupancy of DNMT3A1 and TET1. Indeed, previous studies reported that the genomic binding of TET1 is positively correlated with CpG density [15].

\section{DNMT3A and TET1 binding correlate with histone marks and overall gene expression}

To further understand the impact of DNMT3A and TET1 on the other protein's binding capacity, we looked more closely at different TSS regions and their relationships with the DNMT3B and the DNMT3A isoforms in WT ESCs. First, we clustered all of the TSSs in the mouse genome into five groups based on the binding signals of DNMT3A1, 3A2, and 3B1 (Fig. 4a, b). We focused on Groups 1-3 (G1, G2, and G3) because they exhibited relatively higher occupancy of DNMTs and/or TET1 (Fig. 4a-c). DNMT3A1 specifically binds around the TSSs of G1 genes. In contrast, DNMT3B1 is preferentially enriched in the gene bodies of G2 genes. DNMT3A2 co-binds with DNMT3A1 at G1 genes and together with DNMT3B1 at G2 genes (Fig. 4a, b). Therefore, DNMT3A2 may compensate for the function of DNMT3B1 in Dnmt3b KO cells; however, DNMT3A function cannot be fully compensated by DNMT3B1 in Dnmt3a (both Dnmt3a1 and Dnmt3a2) KO cells, resulting in significant loss of DNA methylation (Fig. 1). Surprisingly, we also found DNMT3A, especially DNMT3A2, binds within the TSSs of G3 genes (Fig. 4a). These results confirmed that DNMT3A and DNMT3B have distinct binding properties and further distinguished the isoform-specific binding patterns of DNMT3A1 and DNMT3A2.

In order to understand how DNMT3A and TET1 influence other epigenetic markers, we examined the histone landscape and gene expression levels in WT cells for each group (Fig. 4c, d). Importantly, we discovered that G1 genes possess high levels of both H3K4me3 and H3K27me3, so called bivalent genes [25], suggesting that DNMT3A1 and TET1 may bind and function together to regulate the expression of bivalent genes in ESCs. These data are also consistent with recent findings that DNMT3A1 shows significant enrichment around bivalent genes [26]. G2 genes exhibit high levels of H3K4me3 and TET1, low levels of H3K27me3 at TSSs and high DNMT3B1 binding in flanking regions; accordingly, they are the most highly expressed gene group in mouse ESCs (Fig. 4c, d). In contrast, G3 genes carry the lowest level of H3K4me3 and TET1, while at the same time their TSSs are bound by DNMT3A and are heavily methylated (Additional file 1: Figure S5), probably 
a

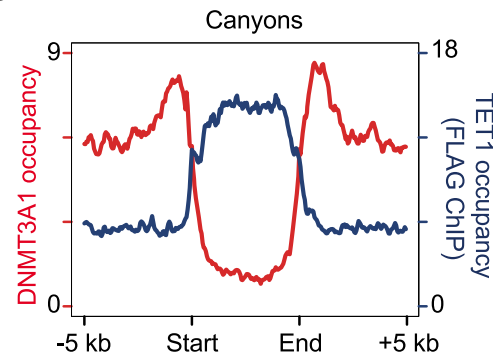

C

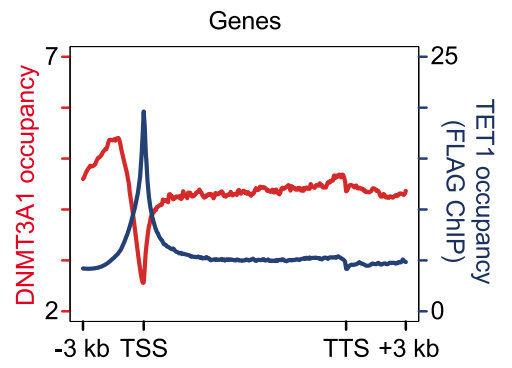

b

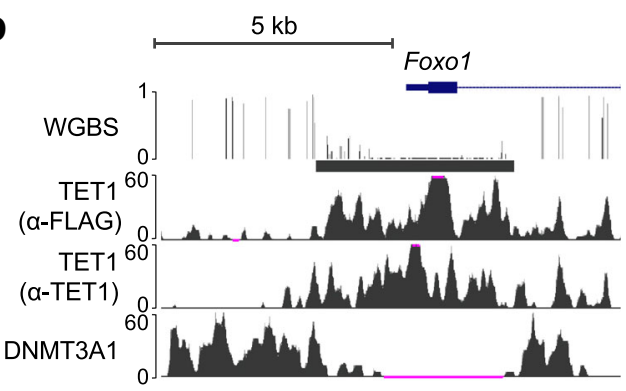

d

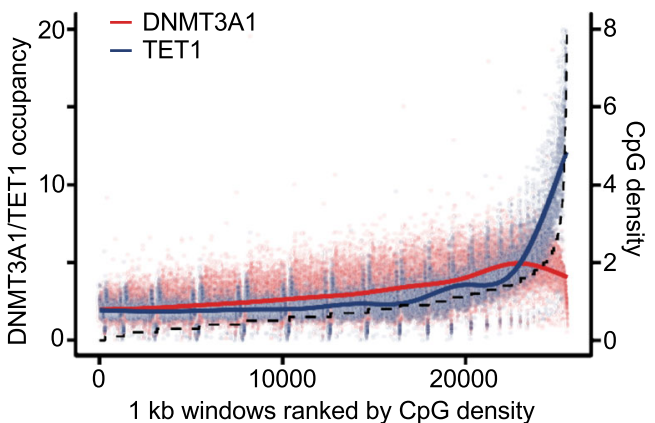

Fig. 3 DNMT3A1 binding is complementary to TET1. a Average binding occupancy of DNMT3A1 and TET1 (anti-FLAG ChIP-seq) across all canyons, normalized to same length (Start-End), and 5-kb flanking regions. b UCSC genome browser track depicts DNA methylation, TET1 (anti-FLAG and anti-TET ChIP), and DNMT3A1 binding profiles around Foxo1 gene promoter in Tet1-FLAG ESCs. c Average binding occupancy of DNMT3A1 and TET1 (anti-FLAG ChIP-seq) across all gene bodies and 3-kb flanking regions. $\mathbf{d}$ DNMT3A1 and TET1 binding enrichment in 1-kb genomic windows ranked by CpG density, which was displayed as a dashed line. The point where DNMT3A and TET1 lines cross on right y-axis represents roughly 1.5 CpG per 100 bp

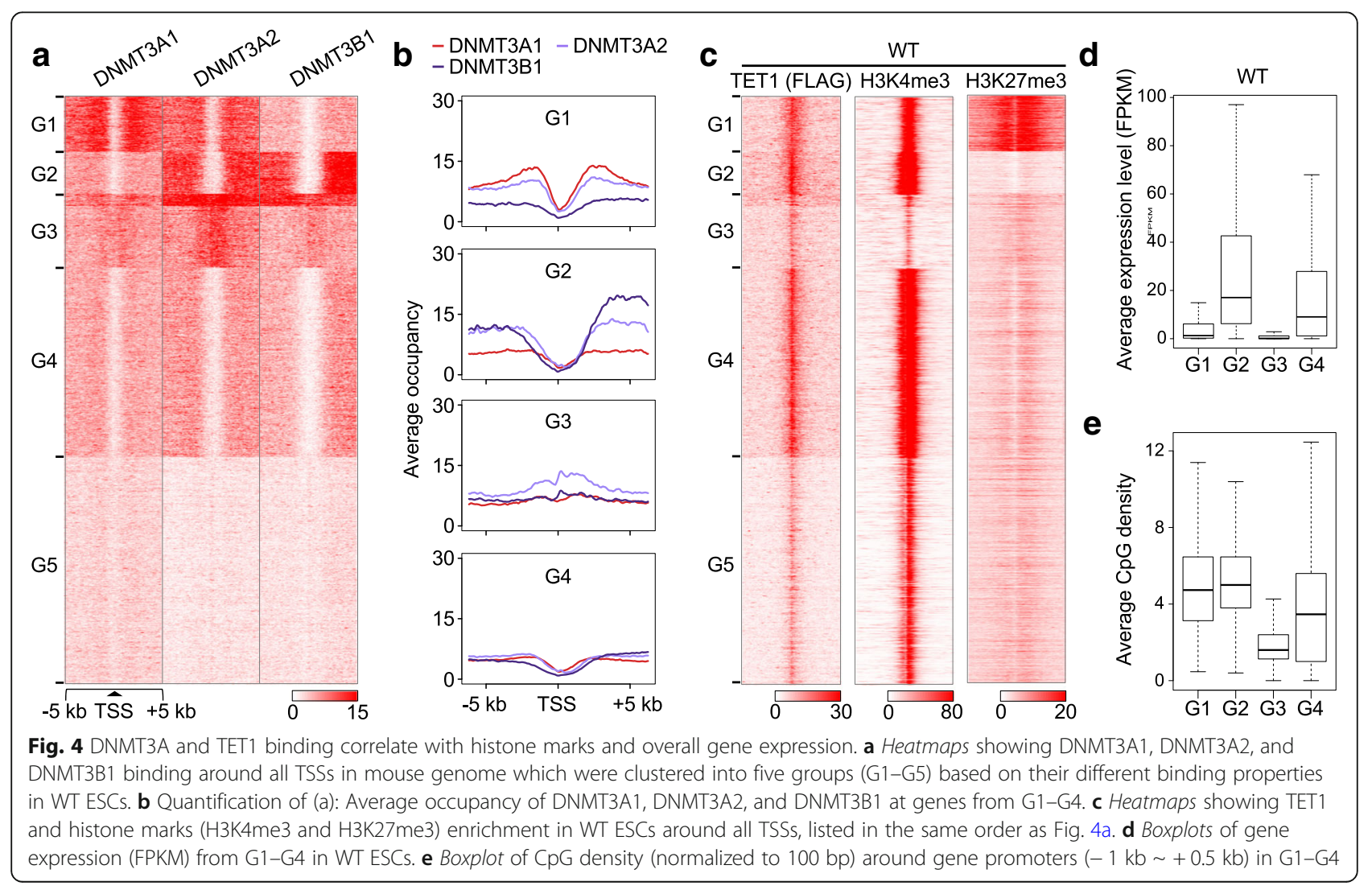


because of lower CpG density at these TSSs [27] (Fig. 4e). Correspondingly, they have the lowest overall expression (Fig. 4d). While the converse of the majority of TSSs, which typically exhibit high TET1 binding, the reciprocal binding pattern of DNMT3A and TET1 is still observed in G3. Thus, the binding pattern of DNMT3A and TET1 correlates very well with histone landscapes and gene expression in WT ESCs.

\section{TET1 protects from DNMT3A1 binding to limit DNA methylation}

The notable exclusion of DNMT3A1 binding to TET1-occupied genomic regions strongly suggests a functional relationship between TET1 and DNMT3A binding to DNA. Previous studies have shown that depletion of TET1 via RNA interference (RNAi) can lead to a slight global increase in the level of $5 \mathrm{mC}$, as well as localized increase in $5 \mathrm{mC}$ at TSSs and the genomic regions flanking their proximal promoters $[15,28]$. To determine the degree to which loss of TET1 permits unabrogated access of DNMT3A1, we generated Bio-Dnmt3a1 - Tet1 KO ES cell lines by utilizing CRISPR/Cas9 and a single guide RNA (sgRNA) previously described [29]. After confirming lack of TET1 protein and induced expression of Bio-DNMT3A1 (Additional file 1: Figure S6a, b), global changes of both $5 \mathrm{mC}$ and $5 \mathrm{hmC}$ were determined in the established cell lines. Dot blot analysis verified increased global $5 \mathrm{mC}$ and decreased $5 \mathrm{hmC}$ (Additional file 1: Figure S6c, d) in Tet1 KO ESCs.

We next determined the distribution of DNMT3A1 in the absence of TET1 by biotin-based ChIP-seq. DNMT3A1 binding capacity increased dramatically around the TET1 binding peaks identified in WT cells (TSS associated), both in overall occupancy (peak height) and by invading into flanking regions (peak width, Fig. 5a). The increased and expanded binding by DNMT3A1 strikingly mirrors the sites of augmented DNA methylation observed in Tet $1 / 2 / 3$ Triple KO (Tet-TKO) ESCs [30] (Fig. 5b). We further examined all TSS regions, comparing DNMT3A1 binding in WT and Tet1 KO ESCs, ranking them by the magnitude of change in DNMT3A1 occupancy after Tet1 KO. The regions with the greatest change were also those with highest TET1 binding in WT ESCs (Additional file 1: Figure S7a). DNMT3A1 binding is also anticorrelated with the extent of H3K4me3 levels (Fig. 4a, c). However, loss of TET1 has little effect on H3K4me3 level at TSSs

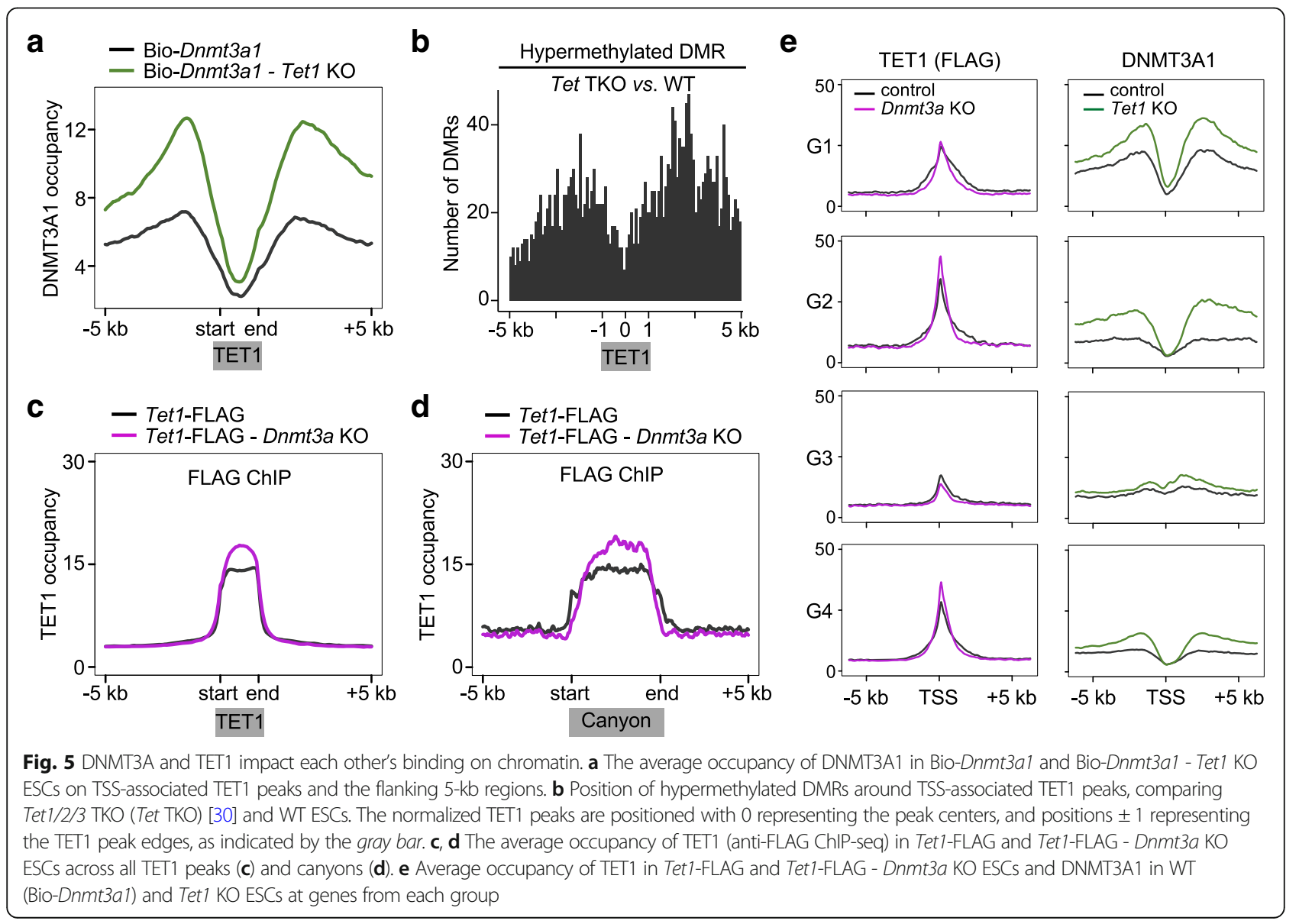


(Additional file 1: Figure S7b, c), making it unlikely that H3K4me3 could impact DNMT3A binding in Tet1 KO ESCs. Collectively, the presence of TET1 constrains DNMT3A1 binding in CpG-rich environments, such as proximal promoter regions, inhibiting DNA methylation.

\section{Dnmt3a ablation has a weak but promotor-specific impact on TET1 distribution}

The finding that TET1 limits DNMT3A's access to TSSs and canyons raised the possibility that DNMT3A may reciprocally constrain TET1. To test this possibility, we deleted Dnmt3a (both Dnmt3a1 and Dnmt3a2) in Tet1-FLAG ESCs (Additional file 1: Figure S7d) using CRISPR/Cas9-facilitated knockout and then performed FLAG ChIP-seq in Dnmt3a KO; Tet1-FLAG ESCs. Similar to the behavior of DNMT3A1 following Tet1 deletion, there was increased TET1 occupancy genome-wide within both TET1 peaks and canyons in Dnmt3a KO cells, compared with the parental Tet1-FLAG ESCs (Fig. 5c, d). However, the magnitude of these changes was modest compared to the experiments described above when Tet1 was deleted. The slightly increased binding of TET1 was limited largely to peak height, with no significant increase in peak width, indicating there was little expansion of access to TET1 following Dnmt3a deletion. The same trend was observed by ChIP-seq with anti-TET1 antibody using the same cells (Additional file 1: Figure S7e, f).

Next, TET1 and DNMT3A1 binding in KO and control cells were displayed based on the same clustering used in Fig. 4 (Additional file 1: Figure S7g and Fig. 5e). Deletion of Tet 1 results in increased binding by DNMT3A1 in all groups, but at a higher level in G1 and G2 genes. On the other hand, deletion of Dnmt3a leads to a moderate increase in binding of TET1 at TSSs of genes from G2 and G4, but a more centralized binding at TSSs from G1 genes, where DNMT3A1 specifically binds (Fig. 4a). Although TET1 binding was affected differently within different groups, it generally increased after Dnmt3a deletion. Nevertheless, the $5 \mathrm{hmC}$ level was lower at genes from all groups, resulting from a significantly reduced level of $5 \mathrm{mC}$ (Additional file 1: Figure S5), the substrate for TET proteins to generate $5 \mathrm{hmC}$ [12].

It has been reported that TET-mediated DNA demethylation mainly occurs at promoters and enhancers [30]. We examined the binding pattern of TET1 at enhancers and surprisingly found that TET1 occupancy at enhancer regions decreased upon Dnmt3a deletion (Additional file 1: Figure S8a), which is distinct from the change at promoters. Furthermore, TET1 abundance is relatively low at enhancers with high levels of H3K27ac and $\mathrm{H} 3 \mathrm{~K} 4 \mathrm{me}$, correlating with lower $\mathrm{CpG}$ density at these stronger enhancers (Additional file 1: Figure S8b).

Together, these data demonstrate the clear reciprocal nature of DNMT3A1 and TET1 binding. While TET1 binding is altered after loss of DNMT3A, it does not extend outside of the canyon boundaries substantially. This suggests TET1 may be restrained within regions of the highest CpG density, thereby allowing it to act as an anchor, impeding access of DNMT3A to limit DNA methylation.

\section{DNMT3A and TET1 dynamically regulate gene expression}

As discussed above, the patterns of histone marks and overall gene expression differ according to DNMT3A and TET1 binding in WT cells. To further elucidate the impact of these proteins on gene expression, we regenerated Dnmt3a KO, Tet1 KO, and Dnmt3a-Tet1 Double KO (DKO) cells from WT J1 ESCs and assessed changes of expression by RNA-sequencing (RNA-seq).

We identified 3007 genes as differentially expressed between WT, Dnmt3a KO, Tet1 KO, and DKO cells. Seven clusters of genes were classified $(\mathrm{C} 1-\mathrm{C} 7)$ based on their expression changes compared to WT. Generally, these differentially expressed genes (DEGs) were either specific to Dnmt3a deletion (C3, C4, and C7) or Tet1 deletion ( $\mathrm{C} 2$ and $\mathrm{C} 6$ ), or changed commonly by both, mostly in the same directions ( $\mathrm{C} 1$ and $\mathrm{C} 5$, Fig. 6a). It was surprising that so few genes were changed in opposing directions in response to Dnmt3a KO or Tet1 KO, considering the opposing enzyme activities (methylation and demethylation) of the two proteins. Compared with WT, many genes (C4, C5, and C7, 39\% of all DEGs) were downregulated in Dnmt3a KO cells, suggesting the correlation between DNA methylation and gene repression is low. Enrichment analysis (Gene Ontology) showed that genes from Hippo and TGF-beta signaling pathway were over-represented in $\mathrm{C} 1$, while PI3K-Akt signaling pathway genes were enriched in $\mathrm{C} 2$ (Additional file 1: Figure S9a). Interestingly, for genes from $\mathrm{C} 1$ and $\mathrm{C} 5$, the expression change caused by Dnmt3a $\mathrm{KO}$ and Tet1 KO was additive, resulting in the greatest bigger change in DKO cells (Fig. 6a). The expression changes of representatives from these pathways was further confirmed by quantitative reverse transcription polymerase chain reaction (RT-qPCR) (Additional file 1: Figure S9b). These data suggest that gene expression in mouse ESCs is dynamically regulated by DNMT3A or/and TET1, and support the concept that DNMT3A and TET proteins can cooperate at specific loci to regulate gene expression [31].

\section{DNMT3A and TET1 constrain expression of bivalent genes, likely via PRC2-mediated H3K27me3}

Next we analyzed the histone marks (H3K4me3 and $\mathrm{H} 3 \mathrm{~K} 27 \mathrm{me} 3$ ) in the various $\mathrm{KO}$ cell lines at differentially expressed genes (Fig. $6 \mathrm{~b}$ and Additional file 1: Figure S10a) and compared them with those of WT cells (Additional file 1: Figure S10b). Remarkably, we found for each cluster of genes, that gene expression 

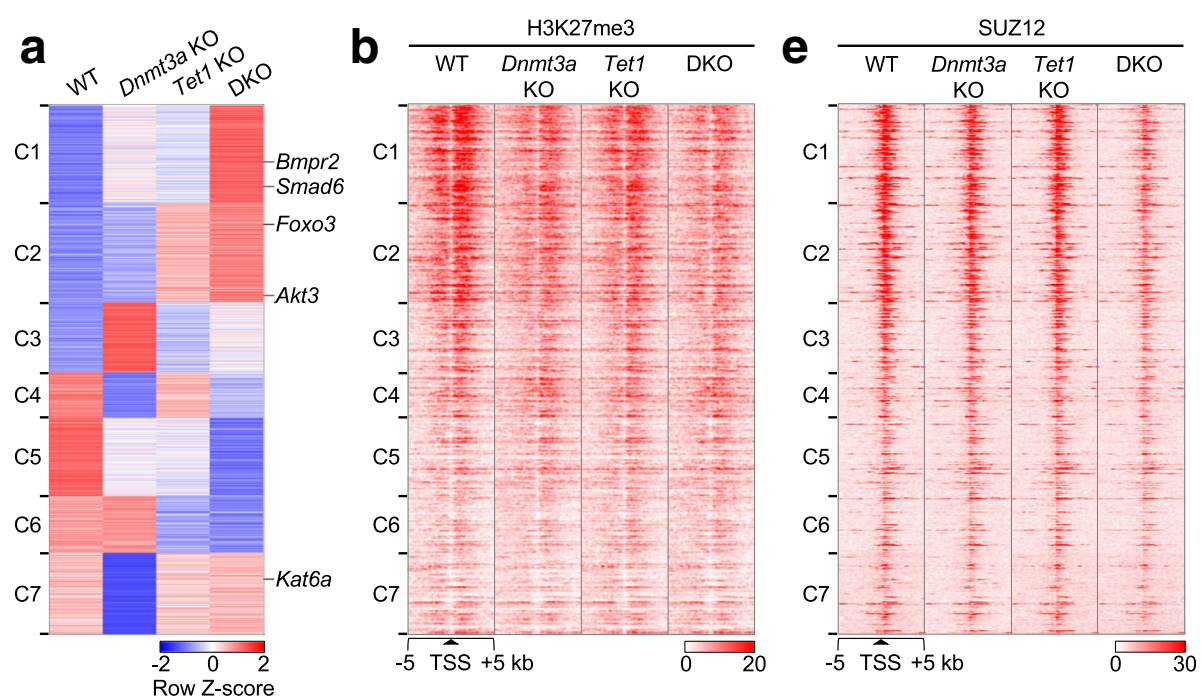

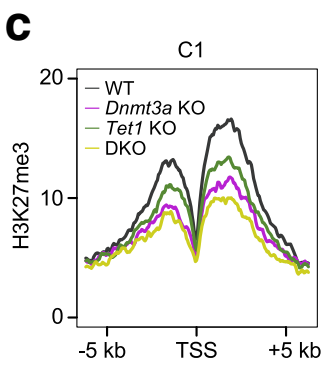

d
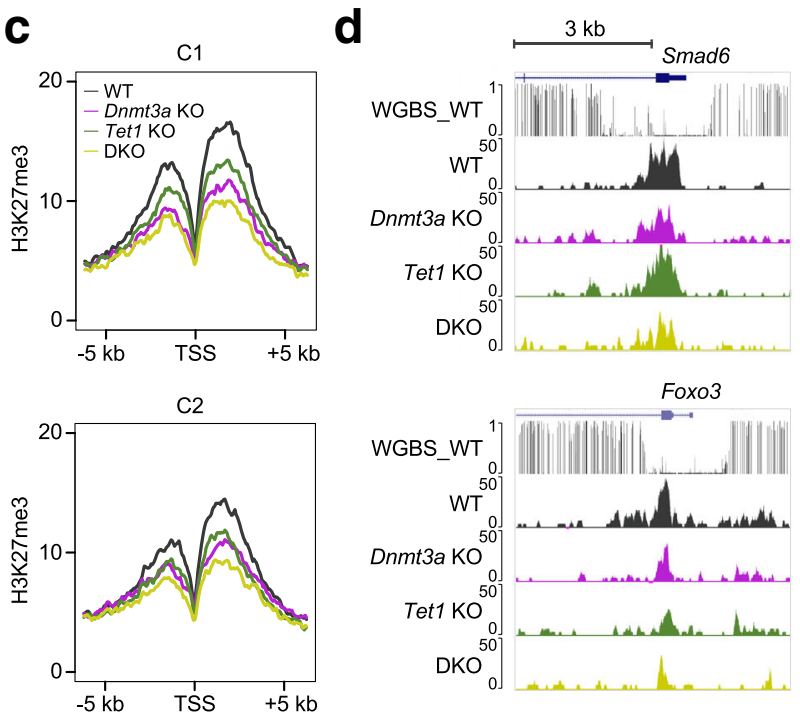
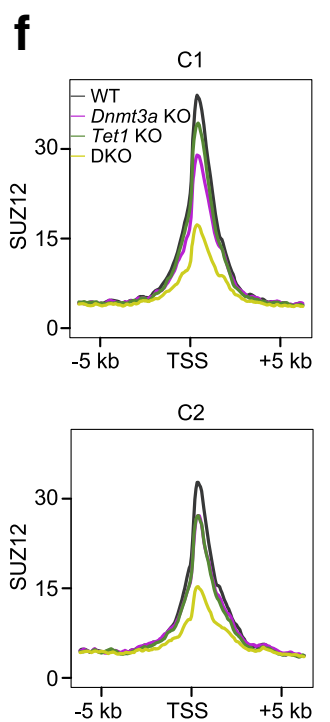

Fig. 6 DNMT3A and TET1 regulate bivalent gene expression via PRC2-mediated H3K27me3. a Hierarchical clustering analysis of differentially expressed genes in WT, Dnmt3a KO, Tet1 KO, and Dnmt3a-Tet1 DKO ESCs. Genes Smad6 and Bmpr2 belong to Hippo / TGF-beta signaling (over-represented in (1) while Foxo3 and Akt3 in PI3K-Akt signaling pathway (over-represented in C2). Their expression difference between cell lines was confirmed by RT-qPCR (shown in Additional file 1: Figure S9b). b Heatmap representations of H3K27me3 enrichment on the flanking $5 \mathrm{~kb}$ surrounding the specific TSSs in each cluster in WT, Dnmt3a KO, Tet1 KO, and DKO ESCs. All TSSs are listed in the same order as Fig. 6a. c Average density profiles for H3K27me3 marks at genes from C1 (top) and C2 (bottom) clusters in indicated cells. $\mathbf{d}$ UCSC genome browser tracks depicting DNA methylation (WGBS) in WT ESCs and H3K27me3 in WT, Dnmt3a KO, Tet1 KO, and DKO ESCs at Smad6 (top) and Foxo3 gene (bottom). e Heatmap representations of SUZ12 occupancy on the flanking 5 kb surrounding the TSSs in cluster C1-C7 in WT, Dnmt3a KO, Tet1 KO and DKO ESCs. $\mathbf{f}$ Average density profiles for SUZ12 occupancy at genes from C1 (top) and C2 (bottom) clusters in indicated cells

changes could be explained by histone modification changes. For example, C3 genes were upregulated in Dnmt3a KO cells in concert with increased H3K4me3. Concomitantly, C7 genes were downregulated along with decreased H3K4me3. C5 genes were downregulated in all $\mathrm{KO}$ cells, accompanied by increased H3K27me3 and decreased H3K4me3 (Additional file 1: Figure S10b). We focused on C1 and C2 because many genes in the two clusters carry both H3K4me3 and H3K27me3 (Additional file 1: Figure
S10a and Fig. 6b). As discussed above, genes in $\mathrm{C} 1$ and C2 are DNMT3A- (especially DNMT3A1) and TET1-specific/enriched bivalent genes (Fig. 4 and Additional file 1: Figure S10c). Loss of DNMT3A and/or TET1 at $\mathrm{C} 1$ genes led to increased gene expression. This upregulation in gene expression was associated with diminished H3K27me3 levels, to a greater extent in Dnmt3a $\mathrm{KO}$ and DKO cells (Fig. $6 \mathrm{a}-\mathrm{c}$ and Additional file 1: Figure S10b), as exemplified by the Smad6 gene (Fig. 6d, Additional file 1: Figure S9b and S10d). Despite similar 
overall changes to histone marks seen at $\mathrm{C} 1$ genes, particularly with respect to $\mathrm{H} 3 \mathrm{~K} 27 \mathrm{me} 3$, expression levels of C2 genes were largely unaffected by Dnmt3a deletion (Fig. 6a-c); this is exemplified by the Foxo3 gene (Fig. 6d, Additional file 1: Figure S9b and S10d).

Because the interplay between DNMT3A and TET1 appeared focused on genes with bivalent histone marks, we examined the impact of DNMT3A and/or TET1 deficiency on the PRC2, which is responsible for establishing the H3K27me3 marks. We checked the expression of Ezh2 and Suz12, two core components of PRC2 complex, and binding activity of SUZ12, comparing Dnmt3a $\mathrm{KO}$, Tet $1 \mathrm{KO}$, and DKO with control cells. No change in expression of these core components was detected in $\mathrm{KO}$ cells (Additional file 1: Figure S10e); however, enrichment of SUZ12 at both $\mathrm{C} 1$ and $\mathrm{C} 2$ genes was reduced, to the greatest extent in DKO cells (Fig. 6e, f and Additional file 1: Figure S10f), which was coincident with the greatest degree of $\mathrm{H} 3 \mathrm{~K} 27 \mathrm{me} 3$ reduction. These results suggest that DNMT3A and TET1 binding at and around specific TSSs functions to mediate binding, and thus overall activity, of the PRC2 complex, ultimately regulating gene expression from bivalent promoters.

To further verify our findings that DNMT3A and TET1 synergistically regulate $\mathrm{H} 3 \mathrm{~K} 27 \mathrm{me} 3$ deposition at bivalent promoters, we performed a normalized histone ChIP-seq by adding spike-in chromatin and antibody in the reactions [32]. Similar results were obtained from the spike-in ChIP-seq: H3K27me3 decreased at DEG clusters $\mathrm{C} 1$ and $\mathrm{C} 2$ in $\mathrm{KO}$ cells, especially DKO cells, while H3K4me3 became lower at C7 genes in Dnmt3a $\mathrm{KO}$ cells (Additional file 1: Figure S11a, b). These results were confirmed by ChIP-qPCR normalized to a positive control in spike-in chromatin (Additional file 1: Figure S11c, d).

\section{DNMT3A and TET1 regulated H3K27me3 enrichment may} be partially DNA methylation-independent

It has been shown that the H3K27me3 mark is lower genome-wide or more broadly distributed in a background with DNA hypomethylation [33, 34]. To examine this here, we performed DNA methylation analysis at single-base resolution in Dnmt3a KO, Tet1 KO, DKO, and WT cells by WGBS. Similar levels of DNA methylation were observed between WT and Tet1 $\mathrm{KO}$ cells and between Dnmt3a KO and DKO cells at different genomic features and DEG clusters (Fig. 7a, b). However, Tet1 KO and DKO cells possessed lower H3K27me3 enrichment (at least at $\mathrm{C} 1$ and C2) compared to WT and Dnmt3a KO, respectively (Fig. $6 \mathrm{~b}$ and Additional file 1: Figure S10b). These data suggest that methylation-independent mechanisms may also be involved in the synergistic regulation of H3K27me3 by DNMT3A and TET1.

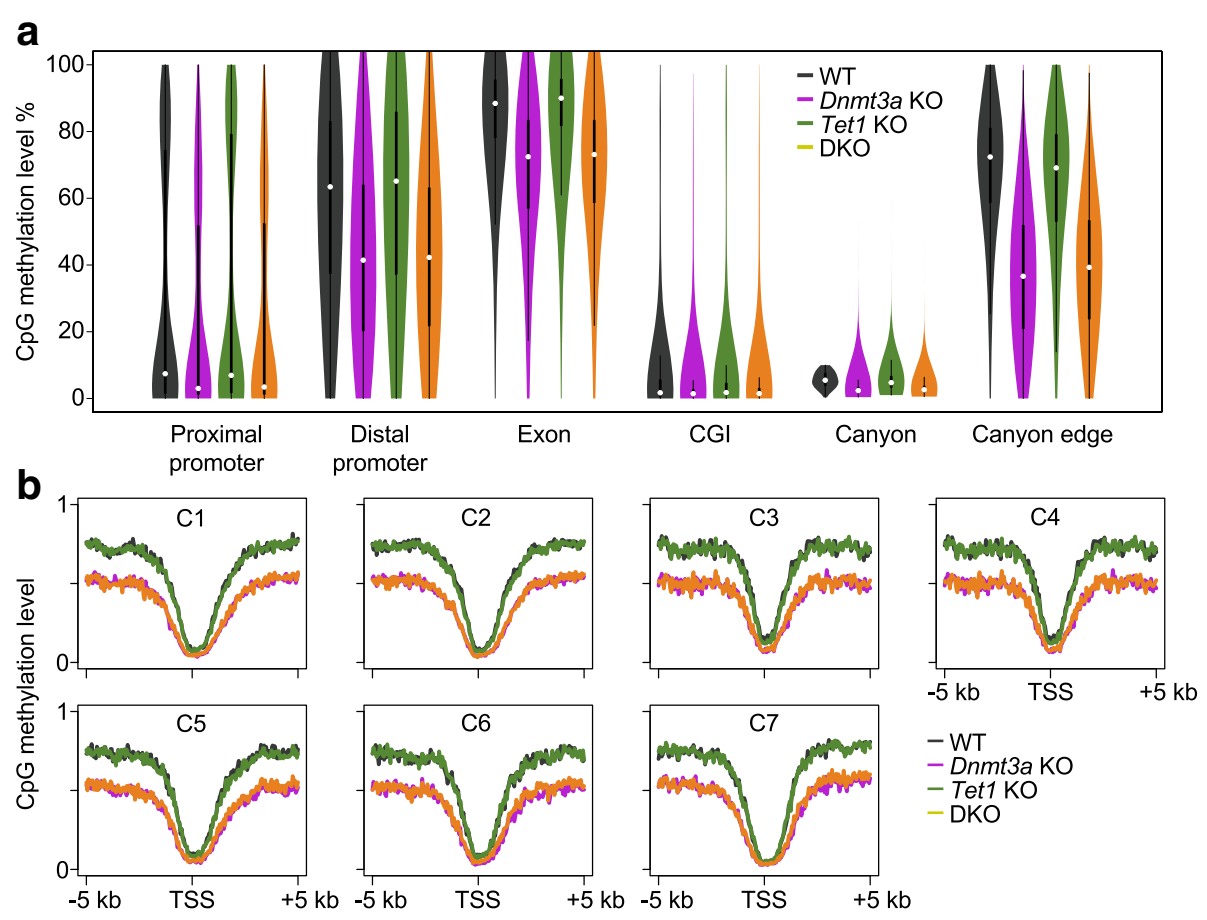

Fig. 7 Analyses of DNA methylation in Dnmt3a KO, Tet1 KO, and DKO ESCs. a Violin plots of CpG methylation levels in WT, Dnmt3a KO, Tet1 KO, and DKO ESCs determined by WGBS at the indicated genomic features. b Average CpG methylation profiles at the flanking 5-kb regions of gene TSSs from clusters C1-C7 (listed in the same order as Fig. 6a) in WT, Dnmt3a KO, Tet1 KO, and DKO ESCs 
Taken together, in this work we showed DNMT3A and TET1 regulate gene expression not only through controlling DNA methylation but also via regulating the histone landscapes at the promoters they bind. These data help resolve the conundrum of the commonly observed lack of correlation between changes in DNA methylation and changes in gene expression by demonstrating that the influence of DNA methylation is mediated proximally via histone mark changes and thus is dependent both on the initial methylation state and the broader epigenetic context.

\section{Discussion}

The maintenance and dynamic regulation of DNA methylation in stem cells and during normal development remains the subject of much debate and research. Here we have demonstrated that DNA methyltransferase DNMT3A and dioxygenase TET1 synergistically regulate gene expression and epigenetic landscapes in mouse ESCs, via their complementary binding profiles and functional interplay.

This study first posits that DNMT3A, as opposed to DNMT3B, is the predominant de novo methyltransferase in establishing and maintaining DNA methylation patterns in mouse ESCs (Fig. 1), similar to its key role in HSCs [9]. Analysis of chromatin binding of DNMTs showed that DNMT3A1 and 3B1 have distinct binding targets, while DNMT3A2 shares binding regions with both $3 \mathrm{~A} 1$ and 3B1. The different binding patterns of DNMT3A isoforms may result from the presence of 219 amino acids at the $\mathrm{N}$-terminus of the longer DNMT3A1 isoform. While DNMT3A and 3B share high homology, and DNMT3B has several splicing isoforms, this $\mathrm{N}$-terminal region is unique to DNMT3A1. The distinct binding pattern also suggests distinct functions in ESC maintenance and differentiation that will be worthy of future investigation. It will also be interesting to determine the presence and role of these isoforms in other tissues, such as the hematopoietic system and skin [35], in which DNMT3A has been shown to have a key functional role.

In addition, our data demonstrate that DNMT3A1 and TET1 have complementary binding patterns at key genomic regions (Fig. 3). Deletion of Tet1 resulted in increased protein accessibility for DNMT3A1 but deletion of Dnmt3a had a modest but varied effect on TET1 binding, suggesting TET1 could serve as an anchor protein to establish boundaries for DNMT3A binding in order to limit DNA methylation (Fig. 5). Even though DNMT3A binding is extended after TET1 loss, it never fully occupies the core regions of promoters with high CpG density where TET1 originally binds. We suspect this is due to the continued presence of other factors, such as transcription factors and histone modifications, which contribute to excluding DNMT3A from occupying those high CpG-dense regions. In contrast, at the centers of TSSs that exhibit lower CpG density, where TET1 and H3K4me3 are absent, DNMT3A does bind. These findings suggest that local CpG density may be a key determinant of the complementary binding patterns between DNMT3A and TET1 (in addition to other factors) and therefore that the epigenetic landscape is encoded in the DNA sequence.

Regarding DNMT and TET interactions, we had previously reported that DNMT3A and TET2 compete and cooperate to regulate HSC functions [31]. Furthermore, during mouse early embryogenesis, the dynamic DNA methylation mediated by TETs and DNMT3s is crucial to regulation of the Lefty-Nodal signaling pathway in body plan formation [36]. Here we bring together these observations by demonstrating that not only DNMT3A (especially DNMT3A1) but also TET1, impacts histone modifications, ultimately influencing gene expression (Fig. 6). As discussed above, they may function together to poise the expression of bivalent genes largely via PRC2-mediated H3K27me3 modification, which is critical for the repression of developmental genes. This concept is also consistent with the reports that loss of DNMT3A and DNMT3B regulates H3K27me3 but has no impact on H3K4me3 [37] and that TET1 is required for chromatin binding of EZH2 in mouse ESCs [15]. This phenomenon may occur during ES cell differentiation: TET1 is gradually lost while DNMT3A is dynamically regulated, affecting $\mathrm{H} 3 \mathrm{~K} 27 \mathrm{me} 3$ and leading to expression of developmental genes. Further investigation is needed to uncover the precise mechanism by which DNMT3A and TET1 influence PRC2 complex activity.

One possibility is a direct protein-protein interaction between DNMT3A/TET1 and PRC components as discussed above. Alternatively, DNMT3A/TET1 may facilitate polycomb enrichment indirectly, through affecting chromatin accessibility. For example, loss of DNMT3A1 could enable local chromatin changes which even modestly permit more transcription factors binding, adjacent to bivalent promoters. Then, such transcription factors would result in reduced polycomb. It will be important to ultimately understand these specific interactions in future studies.

Recently, some of these findings were corroborated using a similar in vivo biotinylation system in murine ES cells [26]. DNMT3A1 was shown to preferentially binds to the edges of $\mathrm{H} 3 \mathrm{~K} 27 \mathrm{me} 3$-rich bivalent $\mathrm{CpG}$ islands, as we see also with canyons. These ESC data are consistent with a model in which DNMT3A1 is mediating turnover of DNA methylation at $\mathrm{CpG}$ island shores in the presence of TET1, indicating a dynamic relationship between the two antagonizing proteins.

While the work here is focused on ESCs, these findings may have relevance for the role of DNMT3A and 
TET2 in hematologic malignancies. Counterintuitively, they have been found co-mutated in some acute myeloid leukemias and T cell lymphoma subtypes [38]. This suggests an oncogenic cooperation between DNMT and TET deficiency that may involve deregulation of DNA methylation and histone modification, specifically at some key loci. Our work could provide additional valuable insights into many of the remaining questions surrounding the role of DNMT and TET proteins in maintaining epigenetic landscapes and gene regulation in a variety of cell types and malignancies.

\section{Conclusions}

Using genetically engineered mouse ESCs as a model, here we reveal the synergistic regulation of epigenetic landscapes by DNMT3A and TET1. We found DNMT3A, especially the longer isoform DNMT3A1, exhibits enrichment at distal promoters and canyon edges, but is excluded from TSSs and canyons where TET1 binds. Knockout experiments suggested that TET1 may play a critical role in defining methylation boundaries by protecting the proximal promoters and canyons from DNMT3A. We demonstrate that DNMT3A and TET1 impact histone modifications which in turn regulate gene expression dynamically. With their binding interplay, they synergistically constrain gene expression from bivalent promoters by regulating PRC2-mediated H3K27me3 enrichment.

\section{Methods}

\section{Cell lines and cell culture}

Mouse ESCs were cultured on $0.1 \%$ gelatin-coated dishes in DMEM (Gibco, 11,965-092) supplemented with 15\% fetal bovine serum (Foundation, 900-108), $1 \times$ non-essential amino acids (Gibco, 11,140-050), $1 \times$ GlutaMAX (Gibco, 35,050-061), $1 \mu \mathrm{M}$ 2-Mercaptoethanol (Sigma, M7522), $1 \times$ sodium pyruvate (Sigma, S8636), $1 \times$ Penicillin/Streptomycin (Gibco, 15,140-122), and $1000 \mathrm{U} / \mathrm{mL}$ LIF (Millipore, ESG1106).

Bio-Dnmt3a1 ESCs were obtained by cloning Dnmt3a1 cDNA (BC007466; Open Biosystems) into pENTR/D-Topo vector (Thermo-Fisher) followed by Gateway recombination into p2Lox and inducible cassette exchange as previously described [23]. The generated cell line expresses an $\mathrm{N}$-terminal Avi-tagged DNMT3A1 under control of a TetO promoter. Biotin tagging was performed in vivo by a ligase BirA encoded from the same vector. CRISPR/Cas9 deletion of Tet1 in this cell line was achieved using pX330 and the guide RNA for exon 4 of Tet1 was previously used [29].

To generate Tet1-FLAG cells, WT J1 ES cells were transfected with $1.0 \mu \mathrm{g}$ eSpCas9-Tet1 [39], $250 \mathrm{ng}$ pRR-puro-Tet1 [40], and $1.5 \mu \mathrm{g}$ donor ssDNA (DNA sequence 5'-3': CAATGTTGTTACCGTGTCCCCATA
CTCTCTCACTCATGTTGCGGGACCCTACAATAGAT GGGTCGGCGGCAGTGGAGACTACAAAGACCATGA CGGTGATTATAAAGATCATGACATCGATTACAAGG ATGACGATGACAAGTAAGTTGGGTCTAAAGGCTT CTCTCATGTAATGCCTTTGCTAATGTGGTGTAGTG GGT) using Lipofectamine 3000 reagent and selected by adding puromycin $(2 \mu \mathrm{g} / \mathrm{mL})$ to the ES medium $24 \mathrm{~h}$ post transfection. After $48 \mathrm{~h}$ of selection, surviving cells were trypsinized and plated at a colony density in normal medium on $10-\mathrm{cm}$ dishes. After one week of culture, individual colonies were picked, expanded, and screened. To further delete Dnmt3a in established Tet1-FLAG ES cell line, exons 18-19 of Dnmt3a (Dnmt3a1) were deleted by dual sgRNAs-facilitated CRISPR/Cas9 system. Guide RNAs were cloned into pX459 vector and transfected into Tet1-FLAG J1 ES cells with Lipofectamine 3000 reagent. The transfected cells were selected, cultured, and screened as above.

Tet1 KO and Dnmt3a-Tet1 DKO J1 ES cells were obtained by deleting exon 4 of Tet 1 alone or together with exons 18-19 of Dnmt3a (Dnmt3a1) using dual sgRNAs-facilitated CRISPR/Cas9. All guide RNAs and genotyping primers used in this paper are listed in Additional file 2: Table S1.

\section{Biotin-streptavidin immunodetection}

After a $16 \mathrm{~h}$ doxycycline $(2 \mu \mathrm{g} / \mathrm{mL})$ induction, whole-cell extracts were obtained from $5 \times 10^{6}$ Bio-Dnmt3a1 ES cells by resuspension in CytoBuster Protein Extraction Reagent (Millipore) followed by 15 -min incubation at room temperature (RT). Non-induced ESCs served as a negative control. Samples were spun at $20,000 \mathrm{~g}, 4^{\circ} \mathrm{C}$ for $15 \mathrm{~min}$ and supernatant was isolated. Immunoprecipitation of biotinylated-DNMT3A1 was performed with M280-streptavidin beads (Thermo Fisher) using whole-cell extract from cultures with or without doxycycline induction. Equal amounts of protein were resuspended in Laemmli buffer and loaded for western blot analysis. The following antibodies were used: anti-DNMT3A (Santa Cruz, sc-20,703), anti-TET1 (provided by $\mathrm{Dr}$. Guoliang $\mathrm{Xu},[24]$ ), and anti- $\beta$-actin (Santa Cruz, sc-47,778).

\section{Chromatin immunoprecipitation}

Biotin-Streptavidin ChIP was performed as described, with some modifications [41]. After a 16-h doxycycline $(2 \mu \mathrm{g} / \mathrm{mL})$ induction, 5 million cells were fixed for $10 \mathrm{~min}$ with $1 \%$ formaldehyde, followed by chromatin extraction and sonication to generate $200-500 \mathrm{bp}$ fragments. Ten percent of chromatin was kept as input. Immunoprecipitation was performed overnight at $4{ }^{\circ} \mathrm{C}$ using M280-streptavidin (Thermo Fisher) magnetic beads. ChIP washes were performed in the following order: $2 \%$ SDS, $1 \times$ high salt buffer $(20 \mathrm{mM}$ Tris $-\mathrm{HCl}$ 
pH 8.0, 0.1\% SDS, 2 mM EDTA, $500 \mathrm{mM} \mathrm{NaCl,} \mathrm{1 \%} \mathrm{Tri-}$ ton $\mathrm{X}-100), 1 \times \mathrm{LiCl}$ buffer (10 mM Tris- $\mathrm{HCl} \mathrm{pH} 8.0,1 \%$ NP40, $1 \mathrm{mM}$ EDTA, $0.25 \mathrm{M} \mathrm{LiCl}, 1 \% \mathrm{NaDOC}), 1 \times \mathrm{TE}$ with $0.2 \%$ Triton $\mathrm{X}-100$, and $1 \times \mathrm{TE}$ buffer. Cross-linking was reversed with the addition of elution buffer $(20 \mathrm{mM}$ Tris- $\mathrm{HCl}, \mathrm{pH}$ 7.5, $5 \mathrm{mM}$ EDTA, $50 \mathrm{mM} \mathrm{NaCl}, 1 \%$ SDS, $50 \mu \mathrm{g} / \mathrm{mL}$ Proteinase K) to washed beads and incubation at $68{ }^{\circ} \mathrm{C}$ with rotation for $3 \mathrm{~h}$. DNA was purified by MinElute PCR purification Kit (Qiagen).

FLAG (Sigma, F1804), TET1 [24], and SUZ12 (Abcam, ab12073) ChIP were performed in a similar procedure with 10 million cells. After incubation of chromatin and Dynabeads Protein A-antibody complex overnight at $4{ }^{\circ} \mathrm{C}$, washes were done in the following order: $1 \times$ low salt buffer $(20 \mathrm{mM}$ Tris- $\mathrm{HCl} \mathrm{pH}$ 8.0, 0.1\% SDS, $2 \mathrm{mM}$ EDTA, $150 \mathrm{mM} \mathrm{NaCl}, 1 \%$ Triton X-100), $2 \times$ high salt buffer (same as above), $2 \times \mathrm{LiCl}$ buffer (same as above), and $1 \times$ TE buffer. Then cross-linking was reversed at $68^{\circ} \mathrm{C}$ and immunoprecipitated DNA was purified by MinElute PCR purification Kit (Qiagen).

H3K4me3 (Millipore, 07-473) and H3K27me3 (Millipore, 07-449; Cell signaling, 9733S) ChIP were performed as previously described [6], with 3 million cells. For ChIP normalization, $50 \mathrm{ng}$ spike-in chromatin (Active Motif, 53,083) and $2 \mu \mathrm{g}$ spike-in antibody (Active Motif, 61,686) were added in each reaction before overnight incubation.

ChIP-seq library preparation and high-throughput sequencing For ChIP-seq, sequencing libraries were prepared using ThruPLEX DNA-seq 48D kit (Rubicon Genomics) following standard protocols. Samples with different dual-index barcodes were combined at equal molar ratios and sequenced as pools. Sequencing of library pools was performed on Illumina HiSeq 2000 or NextSeq 500 system according to Illumina standards, with 50-bp or 75-bp dual-end sequencing. Library de-multiplexing was performed following Illumina standards.

\section{Whole-genome bisulfite library preparation and sequencing} WGBS library construction was done as previously published [6]. For WGBS library construction, $2 \mu \mathrm{g}$ of genomic DNA were isolated from ESCs and fragmented using Covaris S2 sonicator. After DNA fragmentation, libraries were constructed using the Illumina TruSeq DNA sample preparation kit. After ligation, libraries were treated with bisulfite using the EpiTect Bisulfite kit (Qiagen). Ligation efficiency was tested by PCR using TruSeq primers and PfuTurbo Cx Hotstart DNA polymerase (Stratagene). After determining the optimal number of PCR cycles for each sample, a large-scale PCR reaction $(100 \mu \mathrm{L})$ was performed. PCR products were sequenced with Illumina HiSeq sequencing systems.
Anti-cytosine-5-methylenesulfonate-immunoprecipitation, library preparation, and sequencing

Anti-cytosine-5-methylenesulfonate-immunoprecipitation (anti-CMS-IP) was performed as previously described [42] with slight modification. Genomic DNA was fragmented using Covaris Focused-ultrasonicator (average fragment size is $\sim 300 \mathrm{bp}$ ) and ligated with methylated adaptors using NEBNext ${ }^{\oplus}$ Ultra $^{\mathrm{sm}}$ DNA Library Prep Kit for Illumina ${ }^{\circledR}$ following the manufacturer's instructions. The constructed libraries were treated with sodium bisulfite (QIAGEN). Purified bisulfite treated DNA libraries were then denatured for $10 \mathrm{~min}$ at $95^{\circ} \mathrm{C}(0.4 \mathrm{M} \mathrm{NaOH}, 10 \mathrm{mM}$ EDTA) followed by neutralized with the addition of equal volume of ice-cold $2 \mathrm{M}$ ammonium acetate, $\mathrm{pH}$ 7.0. Denatured DNA libraries were incubated with antiserum to CMS in $1 \times$ immunoprecipitation buffer $(10 \mathrm{mM}$ sodium phosphate, $\mathrm{pH}$ 7.0, $140 \mathrm{mM} \mathrm{NaCl}, 0.05 \%$ Triton X-100) overnight at $4{ }^{\circ} \mathrm{C}$ and precipitated with Protein $\mathrm{A}$ and protein G beads. Precipitated DNA was eluted with Proteinase $\mathrm{K}$ and purified by phenol-chloroform extraction. Purified DNA libraries were amplified by 10-12 cycles of PCR using KAPA HiFi HotStart Uracil polymerase. DNA sequencing was carried out using Illumina HiSeq sequencing systems.

\section{Global quantification of $5 \mathrm{mC}$ and $5 \mathrm{hmC}$}

Measurements of $5 \mathrm{mC}$ and $5 \mathrm{hmC}$ were performed by Zymo Research (http://zymoresearch.com) using high-pressure liquid chromatography coupled with mass spectrometry.

Dot blot analysis was performed as previously described [42]. Briefly, purified genomic DNA were denatured in $1 \mathrm{M} \mathrm{NaOH}, 25 \mathrm{mM}$ EDTA at $95^{\circ} \mathrm{C}$ for $10 \mathrm{~min}$ followed by neutralized with ice-cold $2 \mathrm{M}$ ammonium acetate (pH 7.0). The denatured DNA were spotted on a nitrocellulose membrane in a Bio-Dot apparatus (Bio-Rad) with twofold serial dilution. Blotted DNA samples were washed with $2 \times$ SSC buffer under filtered vacuum pressure system and then air-dried at RT for 10-15 min. The DNA were cross-linked with a membrane vacuum baker at $80^{\circ} \mathrm{C}$ for 2 h. Next, the membrane was blocked with 5\% non-fat milk at room temperature for $1 \mathrm{~h}$ and incubated with either anti-5-hmC primary antibody (1:5000, Active Motif, Cat\# 39769) or anti-5mC antibody (1:1000, Millipore, Cat\# MABE146) overnight at $4{ }^{\circ} \mathrm{C}$. The membranes were washed with TBST buffer for 10 min three times then incubated with anti-rabbit secondary antibody (1:10,000, Sigma, Cat\# 7074) for $1 \mathrm{~h}$ at RT. The membranes were then washed for $10 \mathrm{~min}$ three times and visualized by chemiluminescence with West-Q Pico Dura ECL Solution (Gendeport, Barker, TX, USA). To ensure the same loading amount of DNA, after visualization, the same membrane was washed with TBST and stained with $0.02 \%$ methylene blue in $0.3 \mathrm{M}$ sodium acetate ( $\mathrm{pH} 5.2$ ) to ensure equal spotting of total DNA on the membrane. 
Whole genome bisulfite sequencing data analysis We used BSMAP [43] to align the paired-end bisulfite treated reads to the mouse genome $\mathrm{mm} 9$ with the default parameters. BSeQC [44] was then used to remove the technical biases in WGBS data. First, we used the M-bias plot to determine the size of overhang, which would induce the artifact in end-repair process, then remove three bases from the repair end. Second, we removed the clonal reads from the PCR amplification based on a Poisson $P$ value cutoff of $1 \times 10^{-5}$. Third, we excluded one overlapping segment of two read mates to avoid over double counting. After removing the technical biases, we used MOABS [45] to calculate the methylation ratio, identify the hypomethylated regions for one sample and differential methylated regions between paired samples. The methylation ratios were measured as the number of unconverted $\mathrm{CpGs}$ divided by all the covered read numbers for each CpG in the mCall module. The differentially methylated cytosines (DMCs) were defined with the credible difference cutoff 0.2 for CpGs with at least three reads; more than three consecutive CpGs with the same hypo- or hypermethylation state within 300 bp were merged as DMRs in mComp module. The UMRs with methylation ratio $<10 \%$ were detected by using Hidden Markov Model in mOne module. UMRs $<1 \mathrm{~kb}$ in length were excluded in this manuscript. The UMRs in this study were defined as $\geq 1 \mathrm{~kb}$ but $<3.5 \mathrm{~kb}$. UMRs $\geq 3.5 \mathrm{~kb}$ were defined as "canyons."

\section{CMS-seq, histone modification, and TET1 ChIP-seq data analysis}

The raw reads of the 5-hydroxymethylation CMS samples, paired-end $100 \mathrm{bp}$ long, were mapped to the mouse genome mm9 using BSMAP [43]. All the raw reads of histone modification and TET1 ChIP-seq data were mapped to mouse genome mm9 using Burrows-Wheeler Alignment tool (BWA) [46]. Reads that could be mapped to multiple locations were removed. In order to remove the PCR resulted clonal reads, at most two clonal reads were kept for subsequent analysis. The number 2 was based on Poisson $P$ value cutoff of $1 \times$ $10^{-5}$ determined by the total number of reads with respect to the theoretical mean coverage across the genome. The remaining reads were then subject to the module Dtriple in DANPOS v2.2.1 [47] for read depth normalization, input signal subtraction, and the occupancy calculation. The TET1 peaks were called by MACS2 [48] with parameters "broad: True and broad-cutoff: $0.01 . "$

\section{Bio-DNMT3A/3B ChIP-seq data analysis}

The Bio-DNMT3A1, DNMT3A2, and DNMT3B1 samples were sequenced at paired-end $100 \mathrm{bp}$ long. Because several studies have suggested that some DNMT3A binding sites are in repetitive regions, in order to mapping the reads to repetitive regions, the reads were mapped to the mouse genome $\mathrm{mm} 9$ using bowtie [49] by allowing up to two mismatches and 100 alignments for a read. We then used CSEM [50] to allocate the multi-reads using Expectation-Maximization. Similar to other ChIP-seq analysis, we kept two clonal reads to remove the PCR bias. The occupancy of each sample was calculated by the module Dtriple in DANPOS v2.2.1 [47] with the read depth normalization and input signal subtraction.

\section{Dynamic analysis of DNMT3A1, TET1, and histone marks relative to Dnmt3a KO, Tet1 KO or Dnmt3a-Tet1 double KO (DKO)}

We used DANPOS v2.2.1 [47] to quantitatively compare occupancy signal between ChIP-seq samples, with considering the sequencing depth and ChIP-seq input occupancy signal. For H3K4me3 and H3K27me3 spike-in ChIP-seq, we normalized the occupancy signal with factors calculated from Drosophila sequence tags in each sample. DANPOS v2.2.1 calculated the occupancy difference at ten bases between Dnmt3a KO/Tet1 KO and WT. For each ten base pairs, the differential value was measured by the absolute $\log _{10} P$ value. The positive value means the occupancy is greater in Dnmt3a KO/ Tet1 $\mathrm{KO}$ relative to WT, and vice versa.

\section{DNMT3A/3B genome enrichment}

In order to find genome-wide enriched binding sites of DNMT3A/3B, we partitioned the mouse genome to $1-\mathrm{kb}$ windows. First, we removed the windows containing satellite repeats to avoid a false positive caused by the variant repeat number among mouse strains [21]. Furthermore, windows without covered by WGBS data and ChIP-seq input data were removed from subsequent analysis. All the remaining windows were used to identify the statistically enriched regions using the module Dregion in DANPOS v2.2.1 [47]. These enriched regions were used to calculate the distribution of genomic features.

\section{RNA-seq data analysis}

Paired-end reads were sequenced for RNA-seq. We used Trim Galore (http://www.bioinformatics.babraham.ac.uk /projects/trim_galore/) to trim the low-quality bases and the adapters. TopHat [51] was used to mapping the raw reads on mouse genome $\mathrm{mm} 9$ with the default parameters. The gene annotation used for transcriptome alignment is RefSeq downloaded from the UCSC Table Browser (genome.ucsc.edu/cgi-bin/ hgTables?command = start). SAMtools [52] was used in processing the output files of TopHat. Differentially expressed genes were identified by pairwise comparison using the software Cufflinks [51] with cutoff: FDR $\leq 0.05$. Average log- 
transformed gene scaled FPKM expression values of all the differentially expressed genes were used to perform the unsupervised clusters (K-means) and plot the heatmap. Based on the TSS order from the clustering result, we retrieved the normalized occupancy values and the differential values (relative to WT) of TET1, DNMT3A1, H3K27me3, and H3K4me3 across flanking the 5-kb region around gene TSSs, which were then subjected to TreeView to plot the heatmap figures.

\section{Clustering analysis of DNMT3A1, DNMT3A2, and DNMT3B1 occupancy}

In order to measure the different binding properties of DNMT3A1, 3A2, and 3B1 around gene TSSs, we selected the longest isoform for each gene resulting in 22,766 unique gene TSS locations. We then retrieved the normalized occupancy values of DNMT3A1, 3A2, and $3 \mathrm{~B} 1 \mathrm{in} \mathrm{WT}$ across the flanking 5 -kb region around gene TSSs and clustered all TSSs into five groups using the $\mathrm{K}-$ Means $\mathrm{R}$ package. Based on the same order from K-means result, we: (1) retrieved the normalized occupancy values of TET1 (WT and Dnmt3a KO), DNMT3A1 (WT and Tet1 KO), H3K4me3, and H3K27me3 across the flanking 5-kb region around gene TSSs, which were then subjected to TreeView to plot the heatmap figures; (2) calculated CpG density for each gene promoter $(-1 \mathrm{~kb} \sim+0.5 \mathrm{~kb})$; (3) calculated FPKM for each gene from RNA-seq analysis; and (4) plotted the average profiles of $5 \mathrm{mC}$ level and $5 \mathrm{hmC}$ level centered on TSSs.

\section{Additional files}

Additional file 1: Figure S1-S11. Figures and legends for Supplementary Figures S1-S11. (PDF 15.6 Mb)

Additional file 2: Table S1. List of oligonucleotides: guide sgRNAs and primers. (PDF $52 \mathrm{~Kb}$ )

\section{Acknowledgements}

We would like to thank Dr. Guoliang Xu (SIBS, Shanghai) for sharing anti-Tet1 antibody and the Dan L. Duncan Cancer Center (Baylor College of Medicine) Core Grant (CA125123) to support this study.

\section{Funding}

This work was supported by NIH grants: HG007538 and CA193466 (to WL); Al121403 and 1R01Al121403-01A1 (to TC); and DK092883 and CA183252 (to MAG); as well as CPRIT grants: RR140053 (to YH); and CP120348 and CP170002 (to JS). This work was also supported by the American Heart Association (16IRG27250155 to YH) and Texas A\&M University start-up funds (to YH and DS). MJ was supported by 5T32DK60445.

\section{Availability of data and materials}

All datasets generated in this work have been deposited to Gene Expression Omnibus (GEO) under accession number GSE100957, available at https:// www.ncbi.nlm.nih.gov/geo/query/acc.cgi?acc=GSE100957 [53]. Correspondence and requests for materials should be addressed to MAG (goodell@bcm.edu) or WL (wl1@bcm.edu).

\section{Authors' contributions}

Conceptualizations: MAG, TG; Methodology: SMC, XL, and TG; Formal analysis: SMC, XL, and TG; Investigation: SMC, XL, TG, ML MJ, and AG; Resources: TC, MK, and $\mathrm{YH}$; Writing - original draft: SMC and TG; Writing - review and editing: LM, MK, TC, YH, WL, and MAG; Supervision: WL and MAG; Funding acquisition: MJ, JS, YH, DS, TC, WL, and MAG. All authors read and approved the final manuscript.

Ethics approval and consent to participate

Not applicable.

\section{Competing interests}

The authors declare that they have no competing interests.

\section{Publisher's Note}

Springer Nature remains neutral with regard to jurisdictional claims in published maps and institutional affiliations.

\section{Author details}

${ }^{1}$ Stem Cells and Regenerative Medicine Center, Baylor College of Medicine, Houston, TX 77030, USA. ${ }^{2}$ Program in Developmental Biology, Baylor College of Medicine, Houston, TX 77030, USA. ${ }^{3}$ Medical Scientist Training Program, Baylor College of Medicine, Houston, TX 77030, USA. ${ }^{4}$ Division of Biostatistics, Dan L. Duncan Cancer Center, Baylor College of Medicine, Houston, TX 77030, USA. ${ }^{5}$ Department of Molecular and Cellular Biology, Baylor College of Medicine, Houston, TX 77030, USA. ${ }^{6}$ Department of Bioinformatics, School of Life Sciences and Technology, Tongji University, Shanghai, China.

${ }^{7}$ Department of Epigenetics and Molecular Carcinogenesis, The University of Texas MD Anderson Cancer Center, Smithville, TX 78957, USA. ${ }^{8}$ Center for Epigenetics and Disease Prevention, Institute of Biosciences and Technology, Texas A\&M University, Houston, TX 77030, USA. ${ }^{9}$ Lillehei Heart Institute and Department of Pediatrics, University of Minnesota, Minneapolis, MN 55455, USA. ${ }^{10}$ Department of Bioengineering, Stanford University, Stanford, California, USA. ${ }^{11}$ Institute of Biochemistry and Cell Biology, Chinese Academy of Sciences, Shanghai 200031, China.

Received: 16 April 2018 Accepted: 15 June 2018

Published online: 12 July 2018

\section{References}

1. Robertson KD. DNA methylation, methyltransferases, and cancer. Oncogene. 2001;20(24):3139-55

2. Li E, Zhang Y. DNA methylation in mammals. Cold Spring Harb Perspect Biol. 2014;6(5):a019133.

3. Okano M, Bell DW, Haber DA, Li E. DNA methyltransferases Dnmt3a and Dnmt3b are essential for de novo methylation and mammalian development. Cell. 1999;99(3):247-57.

4. Li E, Bestor TH, Jaenisch R. Targeted mutation of the DNA methyltransferase gene results in embryonic lethality. Cell. 1992;69(6):915-26.

5. Tsumura A, Hayakawa T, Kumaki Y, Takebayashi S, Sakaue M, Matsuoka C, et al. Maintenance of self-renewal ability of mouse embryonic stem cells in the absence of DNA methyltransferases Dnmt1, Dnmt3a and Dnmt3b. Genes Cells. 2006;11(7):805-14.

6. Jeong M, Sun D, Luo M, Huang Y, Challen GA, Rodriguez B, et al. Large conserved domains of low DNA methylation maintained by Dnmt3a. Nat Genet. 2014;46(1):17-23.

7. Xie W, Schultz MD, Lister R, Hou Z, Rajagopal N, Ray P, et al. Epigenomic analysis of multilineage differentiation of human embryonic stem cells. Cell. 2013:153(5):1134-48.

8. Meissner A, Mikkelsen TS, Gu H, Wernig M, Hanna J, Sivachenko A, et al. Genome-scale DNA methylation maps of pluripotent and differentiated cells. Nature. 2008;454(7205):766-70.

9. Challen GA, Sun D, Mayle A, Jeong M, Luo M, Rodriguez B, et al. Dnmt3a and Dnmt3b have overlapping and distinct functions in hematopoietic stem cells. Cell Stem Cell. 2014;15(3):350-64.

10. He YF, Li BZ, Li Z, Liu P, Wang Y, Tang Q, et al. Tet-mediated formation of 5carboxylcytosine and its excision by TDG in mammalian DNA. Science. 2011; 333(6047):1303-7.

11. Ito S, Shen L, Dai Q, Wu SC, Collins LB, Swenberg JA, et al. Tet proteins can convert 5-methylcytosine to 5-formylcytosine and 5-carboxylcytosine. Science. 2011;333(6047):1300-3. 
12. Tahiliani M, Koh KP, Shen $Y$, Pastor WA, Bandukwala $H$, Brudno $Y$, et al. Conversion of 5-methylcytosine to 5-hydroxymethylcytosine in mammalian DNA by MLL partner TET1. Science. 2009;324(5929):930-5.

13. Dawlaty MM, Breiling A, Le T, Barrasa MI, Raddatz G, Gao Q, et al. Loss of Tet enzymes compromises proper differentiation of embryonic stem cells. Dev Cell. 2014;29(1):102-11.

14. Williams K, Christensen J, Pedersen MT, Johansen JV, Cloos PA, Rappsilber J, et al. TET1 and hydroxymethylcytosine in transcription and DNA methylation fidelity. Nature. 2011;473(7347):343-8.

15. Wu H, D'Alessio AC, Ito S, Xia K, Wang Z, Cui K, et al. Dual functions of Tet in transcriptional regulation in mouse embryonic stem cells. Nature. 2011; 473(7347):389-93.

16. Dawlaty MM, Ganz K, Powell BE, Hu YC, Markoulaki S, Cheng AW, et al. Tet1 is dispensable for maintaining pluripotency and its loss is compatible with embryonic and postnatal development. Cell Stem Cell. 2011;9(2):166-75.

17. Gu TP, Guo F, Yang H, Wu HP, Xu GF, Liu W, et al. The role of Tet3 DNA dioxygenase in epigenetic reprogramming by oocytes. Nature. 2011 477(7366):606-10.

18. Li Z, Cai X, Cai CL, Wang J, Zhang W, Petersen BE, et al. Deletion of Tet2 in mice leads to dysregulated hematopoietic stem cells and subsequent development of myeloid malignancies. Blood. 2011;118(17):4509-18.

19. Smallwood SA, Kelsey G. De novo DNA methylation: a germ cell perspective. Trends Genet. 2012;28(1):33-42.

20. Chen T, Ueda Y, Dodge JE, Wang Z, Li E. Establishment and maintenance of genomic methylation patterns in mouse embryonic stem cells by Dnmt3a and Dnmt3b. Mol Cell Biol. 2003;23(16):5594-605.

21. Baubec T, Colombo DF, Wirbelauer C, Schmidt J, Burger L, Krebs AR, et al. Genomic profiling of DNA methyltransferases reveals a role for DNMT3B in genic methylation. Nature. 2015;520(7546):243-7.

22. Chen T, Ueda Y, Xie S, Li E. A novel Dnmt3a isoform produced from an alternative promoter localizes to euchromatin and its expression correlates with active de novo methylation. J Biol Chem. 2002;277(41):38746-54.

23. lacovino M, Bosnakovski D, Fey H, Rux D, Bajwa G, Mahen E, et al. Inducible cassette exchange: a rapid and efficient system enabling conditional gene expression in embryonic stem and primary cells. Stem Cells. 2011;29(10):1580-8.

24. Xu Y, Wu F, Tan L, Kong L, Xiong L, Deng J, et al. Genome-wide regulation of $5 \mathrm{hmC}, 5 \mathrm{mC}$, and gene expression by Tet1 hydroxylase in mouse embryonic stem cells. Mol Cell. 2011:42(4):451-64.

25. Bernstein BE, Mikkelsen TS, Xie X, Kamal M, Huebert DJ, Cuff J, et al. A bivalent chromatin structure marks key developmental genes in embryonic stem cells. Cell. 2006;125(2):315-26.

26. Manzo M, Wirz J, Ambrosi C, Villasenor R, Roschitzki B, Baubec T. Isoformspecific localization of DNMT3A regulates DNA methylation fidelity at bivalent CpG islands. EMBO J. 2017;36(23):3421-34.

27. Wu H, Coskun V, Tao J, Xie W, Ge W, Yoshikawa K, et al. Dnmt3a-dependent nonpromoter DNA methylation facilitates transcription of neurogenic genes. Science. 2010;329(5990):444-8.

28. Ito S, D'Alessio C, Taranova OV, Hong K, Sowers LC, Zhang Y. Role of Tet proteins in $5 \mathrm{mC}$ to $5 \mathrm{hmC}$ conversion, ES-cell self-renewal and inner cell mass specification. Nature. 2010;466(7310):1129-33.

29. Wang $H$, Yang H, Shivalila CS, Dawlaty MM, Cheng AW, Zhang F, et al. Onestep generation of mice carrying mutations in multiple genes by CRISPR/ Cas-mediated genome engineering. Cell. 2013;153(4):910-8.

30. Lu F, Liu Y, Jiang L, Yamaguchi S, Zhang Y. Role of Tet proteins in enhancer activity and telomere elongation. Genes Dev. 2014;28(19):2103-19.

31. Zhang $X$, Su J, Jeong M, Ko M, Huang Y, Park HJ, et al. DNMT3A and TET2 compete and cooperate to repress lineage-specific transcription factors in hematopoietic stem cells. Nat Genet. 2016;48(9):1014-23.

32. Egan B, Yuan CC, Craske ML, Labhart P, Guler GD, Arnott D, et al. An alternative approach to ChIP-Seq normalization enables detection of genome-wide changes in histone $\mathrm{H} 3$ lysine 27 Trimethylation upon EZH2 inhibition. PLoS One. 2016;11(11):e0166438.

33. Brinkman AB, Gu H, Bartels SJ, Zhang Y, Matarese F, Simmer F, et al. Sequential ChIP-bisulfite sequencing enables direct genome-scale investigation of chromatin and DNA methylation cross-talk. Genome Res. 2012;22(6):1128-38.

34. Reddington JP, Perricone SM, Nestor CE, Reichmann J, Youngson NA, Suzuki $M$, et al. Redistribution of H3K27me3 upon DNA hypomethylation results in de-repression of Polycomb target genes. Genome Biol. 2013;14(3):R25.

35. Rinaldi L, Datta D, Serrat J, Morey L, Solanas G, Avgustinova A, et al. Dnmt3a and Dnmt3b associate with enhancers to regulate human epidermal stem cell homeostasis. Cell Stem Cell. 2016;19(4):491-501.
36. Dai HQ, Wang BA, Yang L, Chen JJ, Zhu GC, Sun ML, et al. TET-mediated DNA demethylation controls gastrulation by regulating lefty-nodal signalling. Nature. 2016;538(7626):528-32.

37. King AD, Huang K, Rubbi L, Liu S, Wang CY, Wang Y, et al. Reversible regulation of promoter and enhancer histone landscape by DNA methylation in mouse embryonic stem cells. Cell Rep. 2016;17(1):289-302.

38. Couronne L, Bastard C, Bernard OA. TET2 and DNMT3A mutations in human T-cell lymphoma. N Engl J Med. 2012;366(1):95-6.

39. Slaymaker IM, Gao L, Zetsche B, Scott DA, Yan WX, Zhang F. Rationally engineered Cas9 nucleases with improved specificity. Science. 2016; 351(6268):84-8.

40. Flemr M, Buhler M. Single-step generation of conditional knockout mouse embryonic stem cells. Cell Rep. 2015;12(4):709-16.

41. Kim J, Cantor AB, Orkin SH, Wang J. Use of in vivo biotinylation to study protein-protein and protein-DNA interactions in mouse embryonic stem cells. Nat Protoc. 2009:4(4):506-17.

42. Huang Y, Pastor WA, Zepeda-Martinez JA, Rao A. The anti-CMS technique for genome-wide mapping of 5-hydroxymethylcytosine. Nat Protoc. 2012; 7(10):1897-908

43. Xi Y, Li W. BSMAP: whole genome bisulfite sequence MAPping program BMC Bioinformatics. 2009;10:232.

44. Lin X, Sun D, Rodriguez B, Zhao Q, Sun H, Zhang Y, et al. BSeQC: quality control of bisulfite sequencing experiments. Bioinformatics. 2013;29(24):3227-9.

45. Sun D, Xi Y, Rodriguez B, Park HJ, Tong P, Meong M, et al. MOABS: model based analysis of bisulfite sequencing data. Genome Biol. 2014;15(2):R38.

46. Li H, Durbin R. Fast and accurate short read alignment with burrowswheeler transform. Bioinformatics. 2009;25(14):1754-60.

47. Chen $\mathrm{K}, \mathrm{Xi}$ Y, Pan X, Li Z, Kaestner K, Tyler J, et al. DANPOS: dynamic analysis of nucleosome position and occupancy by sequencing. Genome Res. 2013; 23(2):341-51.

48. Zhang Y, Liu T, Meyer CA, Eeckhoute J, Johnson DS, Bernstein BE, et al. Model-based analysis of ChIP-Seq (MACS). Genome Biol. 2008;9(9):R137.

49. Langmead B, Trapnell C, Pop M, Salzberg SL. Ultrafast and memory-efficient alignment of short DNA sequences to the human genome. Genome Biol. 2009;10(3):R25.

50. Chung D, Kuan PF, Li B, Sanalkumar R, Liang K, Bresnick EH, et al. Discovering transcription factor binding sites in highly repetitive regions of genomes with multi-read analysis of ChIP-Seq data. PLoS Comput Biol. 2011;7(7):e1002111.

51. Trapnell C, Roberts A, Goff L, Pertea G, Kim D, Kelley DR, et al. Differential gene and transcript expression analysis of RNA-seq experiments with TopHat and cufflinks. Nat Protoc. 2012;7(3):562-78.

52. Li H, Handsaker B, Wysoker A, Fennell T, Ruan J, Homer N, et al. The sequence alignment/map format and SAMtools. Bioinformatics. 2009:25(16):2078-9.

53. Gu T, et al. The role of DNMT3A and TET1 in regulating promoter epigenetic landscapes. 2018. https://www.ncbi.nlm.nih.gov/geo/query/acc cgi?acc=GSE100957. Accessed 16 May 2018

Ready to submit your research? Choose BMC and benefit from

- fast, convenient online submission

- thorough peer review by experienced researchers in your field

- rapid publication on acceptance

- support for research data, including large and complex data types

- gold Open Access which fosters wider collaboration and increased citations

- maximum visibility for your research: over $100 \mathrm{M}$ website views per year

At BMC, research is always in progress.

Learn more biomedcentral.com/submissions 